\title{
Projecting global mean sea-level change using CMIP6 models
}

\author{
Article \\ Published Version \\ Creative Commons: Attribution 4.0 (CC-BY) \\ Open Access
}

Hermans, T. H. J., Gregory, J. M., Palmer, M. D., Ringer, M. A., Katsman, C. A. and Slangen, A. B. A. (2021) Projecting global mean sea-level change using CMIP6 models. Geophysical Research Letters, 48 (5). e2020GL092064. ISSN 1944-8007 doi: https://doi.org/10.1029/2020GL092064 Available at https://centaur.reading.ac.uk/95974/

It is advisable to refer to the publisher's version if you intend to cite from the work. See Guidance on citing.

To link to this article DOI: http://dx.doi.org/10.1029/2020GL092064

Publisher: American Geophysical Union

All outputs in CentAUR are protected by Intellectual Property Rights law, including copyright law. Copyright and IPR is retained by the creators or other copyright holders. Terms and conditions for use of this material are defined in the End User Agreement.

\section{www.reading.ac.uk/centaur}

\section{CentAUR}

Central Archive at the University of Reading 
Reading's research outputs online 


\section{Geophysical Research Letters}

\section{RESEARCH LETTER \\ 10.1029/2020GL092064}

Key Points:

- The 95th percentile of total sea-leve change projections in 2100 is up to $7 \mathrm{~cm}$ higher for the Coupled Model Intercomparison Project 6 (CMIP6) ensemble than for the CMIP5 ensemble

- The 95th percentile of projected sealevel rise rates near 2100 is up to $21 \%$ larger, implying more pronounced differences beyond 2100

- Depending on climate sensitivity, individual models can project global mean sea-level rise substantially outside the ensemble 5\%-95\% range

Supporting Information:

- Supporting Information S1

Correspondence to:

T. H. J. Hermans,

tim.hermans@nioz.nl

Citation:

Hermans, T. H. J., Gregory, J. M., Palmer, M. D., Ringer, M. A., Katsman, C. A., \& Slangen, A. B. A. (2021).

Projecting global mean sea-level change using CMIP6 models. Geophysical Research Letters, 48, e2020GL092064. https://doi.org/10.1029/2020GL092064

Received 10 DEC 2020 Accepted 31 JAN 2021
(C) 2021. The Authors.

This is an open access article under the terms of the Creative Commons Attribution License, which permits use, distribution and reproduction in any medium, provided the original work is properly cited.

\section{Projecting Global Mean Sea-Level Change Using CMIP6 Models}

\author{
Tim H. J. Hermans ${ }^{1,2}$ (D), Jonathan M. Gregory ${ }^{3,4}$ (D), Matthew D. Palmer ${ }^{3}$ iD,
} Mark A. Ringer ${ }^{3}$ (D), Caroline A. Katsman ${ }^{5}$ (D), and Aimée B. A. Slangen ${ }^{1}$ (D)

${ }^{1}$ Department of Estuarine \& Delta Systems, NIOZ Royal Netherlands Institute for Sea Research, and Utrecht University, Yerseke, The Netherlands, ${ }^{2}$ Department of Geosciences and Remote Sensing, Delft University of Technology, Delft, The Netherlands, ${ }^{3}$ Met Office Hadley Centre, Exeter, Devon, UK, ${ }^{4}$ National Centre for Atmospheric Science, University of Reading, Reading, UK, ${ }^{5}$ Department of Hydraulic Engineering, Delft University of Technology, Delft, The Netherlands

Abstract The effective climate sensitivity (EffCS) of models in the Coupled Model Intercomparison Project 6 (CMIP6) has increased relative to CMIP5. We explore the implications of this for global mean sea-level (GMSL) change projections in 2100 for three emissions scenarios. CMIP6 projections of global surface air temperature are substantially higher than in CMIP5, but projections of global mean thermal expansion are not. Using these projections as input to construct projections of GMSL change with IPCC AR5 methods, the 95th percentile of GMSL change at 2100 only increases by 3-7 cm. Projected rates of GMSL rise around 2100 increase more strongly, though, implying more pronounced differences beyond 2100 and greater committed sea-level rise. Intermodel differences in GMSL projections indicate that EffCS-based model selection may substantially alter the ensemble projections. GMSL change in 2100 is accurately predicted by time-integrated temperature change, and thus requires reducing emissions early to be mitigated.

Plain Language Summary Climate sensitivity measures how much the Earth's surface warms for a given increase in greenhouse gas concentration. In the new generation of global climate models, climate sensitivity has increased. We explore how the simulations of these models affect global mean sea-level (GMSL) rise projections in 2100 for three different emissions scenarios. We compute GMSL projections based on simulated global surface warming (which affects land-ice melt) and thermal expansion of the ocean, using the methods of the fifth Assessment Report of the Intergovernmental Panel on Climate Change. The latest projections of global surface warming are substantially higher than the previous projections, whereas the projections of global thermal expansion are not. Consequently, the upper limits of our GMSL projections increase by 3-7 cm depending on the emissions scenario. This difference will likely become more pronounced beyond 2100. Depending on climate sensitivity, GMSL projections for individual models can differ substantially, implying that using only a subset of models selected based on their climate sensitivity may substantially alter GMSL projections. Since GMSL in 2100 can be predicted well by the cumulative sum of surface warming up to 2100 , it is important to reduce the emission of greenhouse gases early to mitigate GMSL rise.

\section{Introduction}

Global mean sea level (GMSL) has risen by around $1.5 \mathrm{~mm} / \mathrm{yr}$ since the 1960s (Dangendorf et al., 2017; Frederikse et al., 2018) and is projected to rise at an accelerated pace unless greenhouse gas emissions are substantially reduced (Oppenheimer et al., 2019). Limiting the adverse impacts of sea-level rise (Nicholls \& Cazenave, 2010) requires accurate sea-level projections to underpin effective coastal decision making and adaptation planning. GMSL change results from both ocean density changes (steric sea-level change) and the exchange of mass (barystatic sea-level change) between ocean and cryosphere or land. Global mean steric sea-level change is nearly equal to global mean thermal expansion (GTE), since global mean halosteric change is negligible (Gregory et al., 2019; Gregory \& Lowe, 2000).

Ensembles of complex, coupled global climate models (GCMs), such as the models in the fifth phase of the Coupled Model Intercomparison Project (CMIP5, Taylor et al., 2012), form the basis of many GMSL projections (e.g., Church et al., 2013; Kopp et al., 2014; Oppenheimer et al., 2019; Palmer et al., 2020; Palmer 
et al., 2018; Slangen et al., 2014, 2012). The fifth Assessment Report of the Intergovernmental Panel on Climate Change (IPCC AR5) constructed a Monte Carlo ensemble of GMSL projections from CMIP5 simulations of GTE and global mean surface air temperature (GSAT), with GSAT-driven parameterizations of the surface mass balance contributions to land-based ice melt (Church et al., 2013). Several subsequent studies built on this approach, updating the contribution of Antarctic ice sheet dynamics based on post-AR5 modeling studies (Oppenheimer et al., 2019; Palmer et al., 2018, 2020).

The GSAT response to radiative forcing varies across GCMs according to the strength of different climate feedbacks, and can be characterized by estimates of climate sensitivity. In particular, equilibrium climate sensitivity (ECS) measures the GSAT response in GCMs upon reaching equilibrium following an instantaneous doubling of $\mathrm{CO}_{2}$ concentration in the atmosphere. Since running coupled GCMs to equilibrium is computationally very expensive, ECS is often approximated with effective climate sensitivity (EffCS). EffCS is obtained through linearly regressing the top-of-atmosphere radiative flux anomaly against GSAT in idealized experiments in which a GCM has not yet reached equilibrium (Gregory et al., 2004).

The current generation of GCMs in CMIP6 (Eyring et al., 2016) has a positively skewed EffCS distribution, including multiple models with a higher EffCS than the CMIP5 range (2.1-4.7 K; Andrews et al., 2012) and the 5\%-95\% range constrained by multiple lines of evidence (2.3-4.7 K; Sherwood et al., 2020). Consequently, CMIP6 models project a larger GSAT increase for a given emissions scenario (Forster et al., 2020). This has sparked an important debate about what model developments have caused the increase in EffCS and whether these developments have improved model physics. The increased EffCS in CMIP6 has been attributed to stronger positive cloud feedback (Meehl et al., 2020; Zelinka et al., 2020), and the representation of clouds has improved in several CMIP6 models compared to CMIP5 (e.g., Bjordal et al., 2020; Bodas-Salcedo et al., 2019; Gettelmann et al., 2019; Swart et al., 2019; Voldoire et al., 2019; Williams et al., 2020). On the other hand, some high-EffCS CMIP6 models overestimate historical warming, suggesting their future warming may be too large (Brunner et al., 2020; Forster et al., 2020; Nijsse et al., 2020; Tokarska et al., 2020). Still, an EffCS higher than $5 \mathrm{~K}$ cannot be discounted as physically implausible (Bjordal et al., 2020). Thus, investigating the consequences of increased EffCS in CMIP6 for projected climate change is important.

Although multiple studies have investigated the consequences of increased EffCS in CMIP6 for GSAT change projections, the consequences for GMSL change projections have only been studied using a reduced complexity model (Vega-Westhoff et al., 2020). Here, we generate GMSL change projections based on CMIP6 using the Monte Carlo approach of IPCC AR5 (Church et al., 2013), including both GTE and the GSAT-driven barystatic contributions, and compare these to CMIP5-based projections. Isolating the impact of CMIP6 simulations using consistent methods is an important step to ensure traceability to past IPCC projections of global and regional sea-level change. Given the complex correlations among different GMSL change components (Palmer et al., 2020), increased EffCS may not simply lead to increased GMSL projections. Additionally, the increased EffCS in CMIP6 motivates us to premise high-risk, low-probability GMSL projections on individual models, exploring high-end projections to a fuller extent than was previously possible with CMIP5.

In Section 3.1, we compare CMIP5 and CMIP6 projections of GTE and GSAT change, which form the input to the GMSL projections. Next, we investigate their relationship with EffCS in Section 3.2. We include their relationship with the transient climate response (TCR), another policy-relevant metric of climate sensitivity, in the supporting information. In Section 3.3, we compare CMIP5- and CMIP6-based GMSL projections and show GMSL projections based on individual CMIP6 models. Since we focus on the impact of increased EffCS on interensemble and intermodel uncertainties using IPCC AR5 methods, we acknowledge but do not consider more recent insights into the potential instability of the Antarctic ice sheet (e.g., DeConto \& Pollard, 2016; Edwards et al., 2019).

\section{Data and Methods}

\subsection{CMIP5 and CMIP6 Model Data}

We use monthly CMIP5 GTE and GSAT data ("zostoga" and global mean "tas" variables) from IPCC AR5 (Church et al., 2013) for representative concentration pathways (RCPs, Meinshausen et al., 2011) 8.5, 4.5, 
and 2.6. This ensemble contains 21 models from 13 modeling centers. For three models RCP2.6 data was not provided and instead emulated by IPCC AR5 using the simple climate model of Good et al. (2011, 2013).

The CMIP6 data were downloaded from the Earth System Grid Federation (ESGF) for three shared socioeconomic pathways (SSPs, O'Neill et al., 2014). We picked SSPs with high fossil-fueled, middle-of-the-road and sustainable-development narratives, with nameplate radiative forcing in 2100 approximately corresponding with the aforementioned RCPs: SSP5-RCP8.5, SSP2-RCP4.5 and SSP1-RCP2.6, respectively. For CanESM5 alone, both RCP- and SSP-forced simulations were available from ESGF. The median GSAT change in 20812100 (relative to 1986-2005) of the 5 RCP-forced and 25 SSP-forced variants of CanESM5 differ by 0.27, 0.19, and -0.15 K (SSP minus RCP) for SSP5-RCP8.5, SSP2-RCP4.5 and SSP1-RCP2.6, respectively. These differences will likely reduce in the multimodel mean, so based on this preliminary comparison we assume that differences in GSAT change between CMIP5 and CMIP6 are mainly due to increased EffCS (following Forster et al., 2020). Differences between RCPs and SSPs may have a larger relative effect on GTE because the interensemble differences in GTE are smaller (Section 3.1).

Our CMIP6 ensemble includes 20 models from 12 modeling centers (Table S1). For these models both GTE and GSAT were available by November 2, 2020, for the historical simulation (1850-2014) and all three emissions scenarios (2015-2100). We select the first available simulation variant (identified by "ripf”, Table S1) of each model. Additionally, we require models to provide fully overlapping preindustrial control runs for GTE to allow us to correct for model drift, and idealized experiments to allow us to compute EffCS and TCR (see Section 2.2). All CMIP6 GCMs providing GSAT but not necessarily GTE ( $\mathrm{n}=31$, Table S1) are used for context in Figures S2, S3 and Table S3.

We corrected GTE for model drift, which can arise from the slow adjustment of the deep ocean and/or imperfect representation of energy conservation in the model simulations (Hobbs et al., 2016; Sen Gupta et al., 2013). To remove drift, we apply a least squares quadratic fit to the full control experiment of each model and subtract the overlapping part of the fit from the historical and scenario runs. Although we chose a quadratic fit for consistency with IPCC AR5 (Church et al., 2013), the drift is nearly linear for most CMIP6 models (Figure S1). This is consistent with the analysis of Hobbs et al. (2016) for CMIP5 models. The difference between linear and quadratic drift-correction (2081-2100 minus 1986-2005) is largest for INM-CM5-0 $(0.32 \mathrm{~cm})$, which is small compared to projected GTE $(8-37 \mathrm{~cm}$; Figure 1). Finally, the monthly GTE and GSAT data were annually averaged.

\subsection{Calculating EffCS and TCR}

CMIP5 EffCS and TCR were obtained from Andrews et al. (2012) and Forster et al. (2013), respectively. For CMIP6, we used the same method to compute these (see Table S1). To obtain EffCS, for each GCM we linearly regress the top-of-atmosphere radiative flux anomaly $\Delta N$ against GSAT in 150 years of abrupt-4xCO experiments (Gregory et al., 2004). We use the $\mathrm{CO}_{2}$ quadrupling instead of doubling experiment, because the signal-to-noise ratio is higher. Additionally, we assume that $4 \mathrm{xCO}_{2}$ forcing is twice $2 \mathrm{xCO}_{2}$ forcing and that the climate feedback parameter is constant. Extrapolating $\Delta N$ to zero to approximate equilibrium, EffCS is found as 0.5 times the x-intercept. EffCS underestimates ECS in GCMs because the net climate feedback becomes less effective at restoring radiative equilibrium over time (Andrews et al., 2012; Rugenstein et al., 2020). Nevertheless, EffCS is a widely used metric relevant to 21st-century climate change (Grose et al., 2018) and thus a useful basis of comparison between CMIP5 and CMIP6. To obtain TCR, we calculate the mean GSAT change in years $61-80$ in $1 \% \mathrm{CO}_{2}$ experiments (window centered around the time of $\mathrm{CO}_{2}$ doubling). For MIROC-ESM-CHEM (CMIP5) these idealized experiments were not available.

\subsection{GMSL Projection Methodology}

To ensure traceability to previous GMSL projections, we compute CMIP6-based projections using the same approach as in IPCC AR5 (Church et al., 2013). For each emissions scenario, we generate a 450,000-member Monte Carlo ensemble of GMSL projections. These projections contain contributions of thermal expansion, land-ice mass changes and land-water storage changes. The inputs to these projections are the 
(a) SSP5-RCP8.5

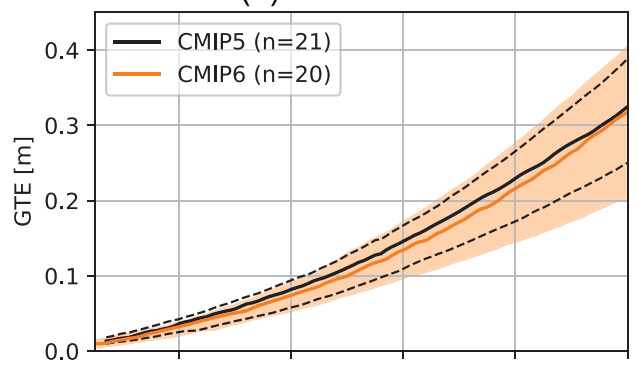

(b) SSP2-RCP4.5

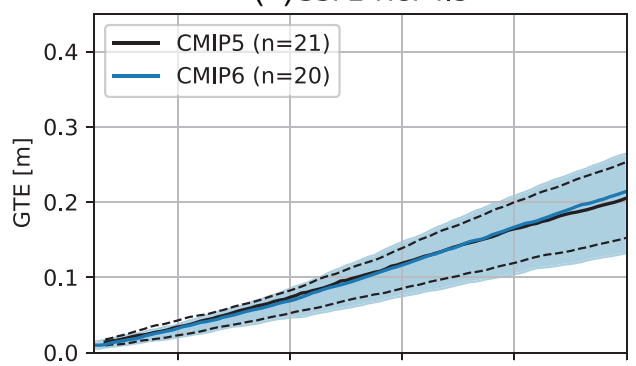

(c) SSP1-RCP2.6

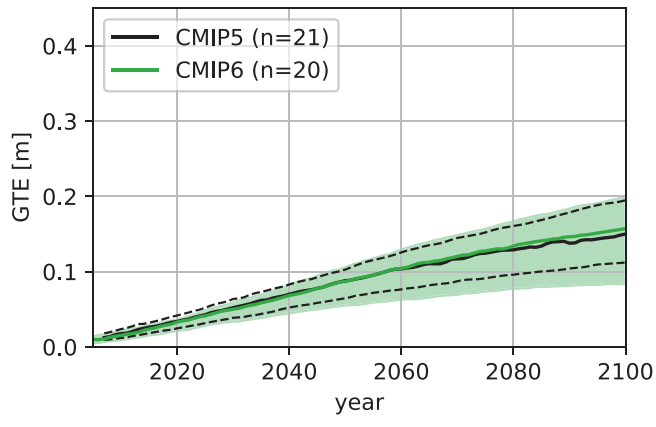

(d) SSP5-RCP8.5

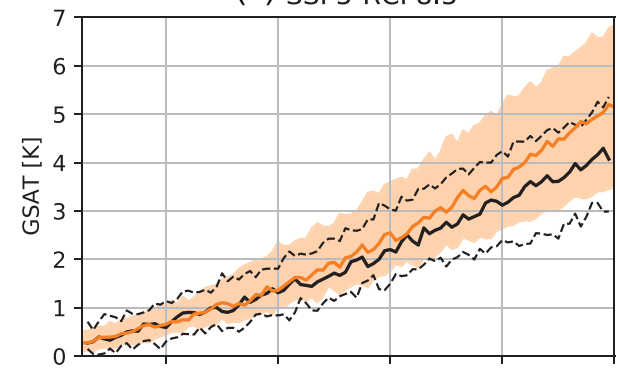

(e) SSP2-RCP4.5

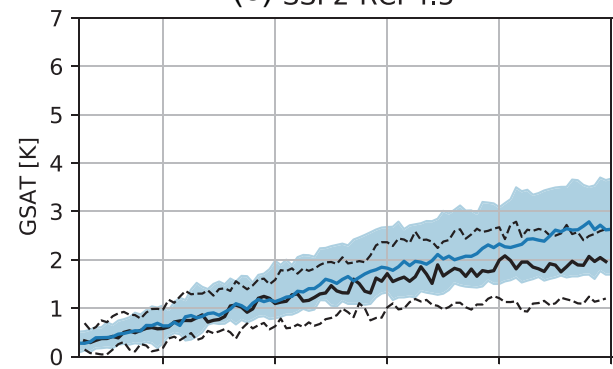

(f) SSP1-RCP2.6

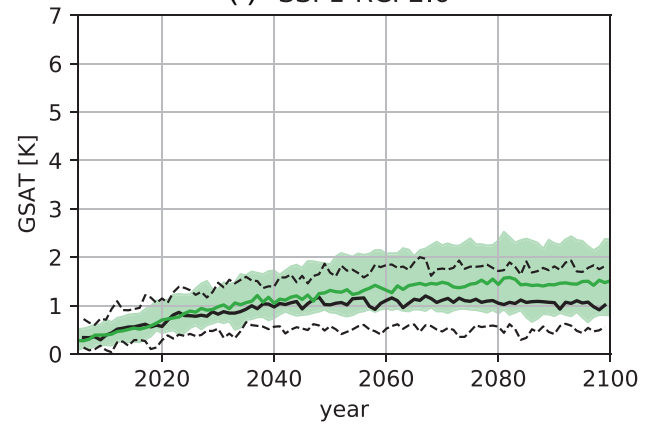

Figure 1. Ensemble median (CMIP6: colored solid lines; CMIP5: black solid lines) and 5\%-95\% range (CMIP6: colored shaded area; CMIP5: black dashed lines) of (a-c) GTE (m) and (d-f) GSAT(K) change of the 20-member CMIP6 and 21-member CMIP5 ensembles, relative to the 1986-2005 average, for SSP5-RCP8.5 (orange, (a, d)), SSP2-RCP4.5 (blue, (b, e)), and SSP1-RCP2.6 (green, (c, f)). CMIP: Coupled Model Intercomparison Project; GSAT: global mean surface air temperature; GTE: global mean thermal expansion; SSP: shared socioeconomic pathway; RCP: representative concentration pathway.

time-dependent CMIP6 mean and standard deviation of GTE and GSAT change, from which 450 samples are drawn (see Text S1 and flowchart therein for details). Through parameterization schemes (Text S1), the contribution of glaciers and the surface mass balance of the Greenland and Antarctic ice sheets depend on GSAT change, while the other barystatic contributions depend only on time. For comparison with the projections of Palmer et al. (2020), and indirectly with the projections of Oppenheimer et al. (2019), we also use an alternative parameterization of Antarctic dynamical ice discharge based on projections of Levermann et al. (2014).

For each GTE and GSAT sample pair, 1,000 samples are generated to represent methodological uncertainty in the parameterization schemes. This results in an ensemble of GMSL projections with $450 \times 1,000=450,000$ members. These projections converge with a $0.01 \mathrm{~m}$ uncertainty for GMSL change and its components and $0.1 \mathrm{~mm} / \mathrm{yr}$ for its rate (Church et al., 2013). Thus, subsequently computed GMSL projections can differ randomly by these amounts. We present a flowchart and a summary of the methods in Text S1, and refer to Church et al. (2013) and Palmer et al. (2020) for further information.

Finally, we also derive GMSL projections based on the GTE and GSAT simulations of each of the 20 CMIP6 models individually (Text S1). This results in 20 ensembles of 1,000 members each, which allow us to study 
intermodel uncertainty related to EffCS. Additionally, equally weighting all models these ensembles can be joined into a 20,000-member ensemble to test the sensitivity to the assumptions made in constructing the 450,000-member ensemble (Section 3.3).

\section{Results}

\subsection{Comparing CMIP5 and CMIP6 Ensemble Projections of GTE and GSAT Change}

We first compare CMIP5 and CMIP6 GTE and GSAT change projections, which form the input to the GMSL projections in Section 3.3. Despite increased EffCS in CMIP6, the CMIP5 and CMIP6 ensemble means and medians of GTE, averaged over the period 2081-2100, are similar (Figures 1a-1c \& key statistics in Table S2). The 5\%-95\% range has widened from CMIP5 to CMIP6, particularly toward lower values, mainly due to the GTE projections of INM-CM4-8 (EffCS = 1.82 K) and INM-CM5-0 (EffCS = 1.92 K). Compared to CMIP5, the 95th percentiles of CMIP6 GTE increase by $1 \mathrm{~cm}(+4 \%,+5 \%$, and $+3 \%)$ under SSP5-RCP8.5, SSP2-RCP4.5, and SSP1-RCP2.6, respectively. The fifth percentiles of GTE correspondingly decrease by 4, 2 , and $2 \mathrm{~cm}(-17 \%,-13 \%$ and $-22 \%)$. However, a two-sided Kolgomorov-Smirnov test suggests we cannot reject the null hypothesis that CMIP5 and CMIP6 GTE are drawn from the same underlying distribution $(p \geq 0.05$, Figure S2).

In contrast to GTE, the mean, median, and 5\%-95\% range of CMIP6 GSAT change at the end of the 21st century are substantially higher than in CMIP5 for all emissions scenarios (Figures 1d-1f \& Table S2). The CMIP6 medians, averaged over 2081-2100, are 0.80, 0.60 and $0.43 \mathrm{~K}$ higher than for CMIP5 for SSP5RCP8.5, SSP2-RCP4.5, and SSP1-RCP2.6, respectively $(+22 \%,+31 \%$, and $+41 \%$; the relative difference due to increased EffCS could be larger in stabilization scenarios because the ocean heat uptake (OHU) efficiency declines faster than in higher emissions scenarios). The 95th percentile changes increase by $1.36,0.91$, and $0.55 \mathrm{~K}(+29 \%,+35 \%$, and $+31 \%)$. The CMIP5 and CMIP6 distributions of GSAT change are only statistically different for SSP2-RCP4.5 ( $<<0.05$, Figure S2). However, this does not mean that the interensemble differences for SSP5-RCP8.5 and SSP1-RCP2.6 are unimportant.

\subsection{Relation of GTE and GSAT Change with Climate Sensitivity}

The 20-member CMIP6 ensemble has a higher median EffCS and TCR than the CMIP5 ensemble (4.04 and $2.00 \mathrm{~K}$ compared to 3.50 and $1.88 \mathrm{~K}$, respectively), and spans a larger range (e.g., EffCS of 1.83-5.62 K compared to 2.08-4.67 K; Table S2). However, insufficient evidence exists for significant statistical difference between the CMIP5 and CMIP6 distributions (Figure S3). Nevertheless, the higher upper tail of CMIP6 motivates investigating the differences further.

To interpret the interensemble differences in GTE and GSAT change given the increased climate sensitivity in CMIP6, we linearly regress GTE and GSAT change in 2081-2100 against EffCS (Figure 2). We find statistically significant linear relationships with EffCS for both GTE (Figures 2a-2c) and GSAT change (Figures 2d-2f), except for CMIP5 GTE under SSP1-RCP2.6 (Figure 2c). Thus, EffCS is a relevant metric for 21st-century climate change. However, since EffCS represents the GSAT response to $\mathrm{CO}_{2}$-doubling, it explains much but not all of the intermodel variance of GSAT change in emissions scenarios with multiple forcing agents (Figures $2 \mathrm{~d}-2 \mathrm{f}, R^{2}$ values). The role of OHU in determining the tendency of the curve of GSAT against EffCS to flatten for increasing EffCS likely causes the non-zero y-intercepts.

Linear regression against EffCS explains much less of the variance of GTE than of GSAT change (10\%-63\% vs. 59\%-91\%; Figure 2). While GTE correlates positively with GSAT change ( $r=0.59-0.80$; Figure S5), GTE is also controlled by the ocean dynamics governing OHU and expansion efficiency, which cause intermodel spread (Melet \& Meyssignac, 2015). For example, OHU in CMIP6 models could partially occur in regions contributing little to increased GSAT change, or be driven by wind-driven subduction unrelated to GSAT change. Aspects like these could explain why GTE projections do not necessarily increase from CMIP5 to CMIP6, despite increased EffCS.

For both GTE and GSAT change, the correlations with EffCS are higher for higher emissions scenarios (Figure 2), consistent with previous results for GSAT change in CMIP5 (Grose et al., 2018). The reason is likely the larger ratio of forced signal to internal variability for higher emissions scenarios (Lyu et al., 2015). 
(a) SSP5-RCP8.5

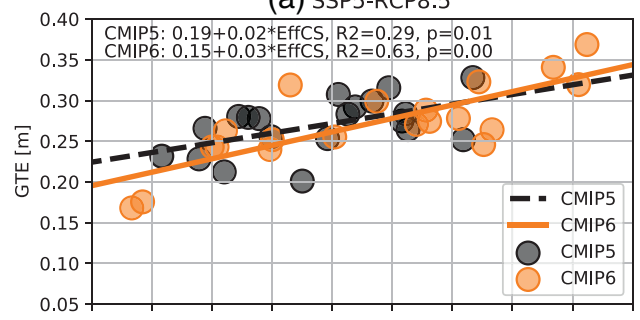

(b) SSP2-RCP4.5

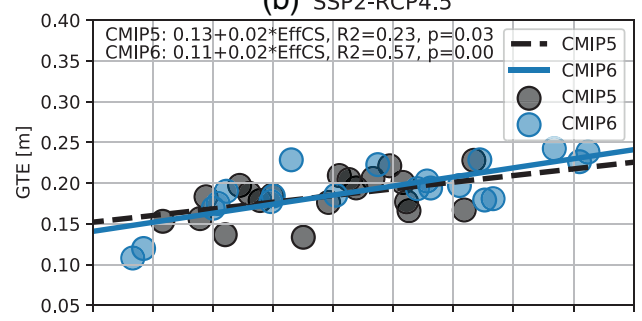

(c) SSP1-RCP2.6

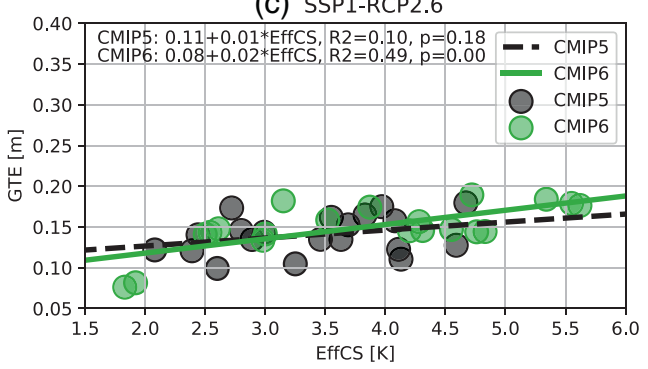

(d) SSP5-RCP8.5

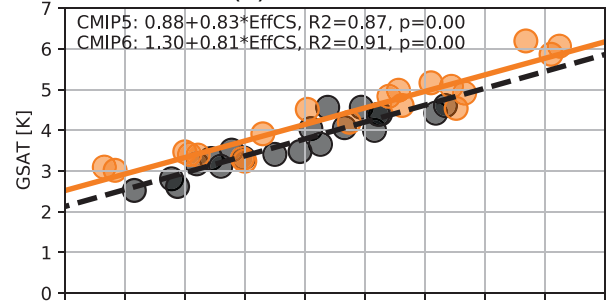

(e) SSP2-RCP4.5

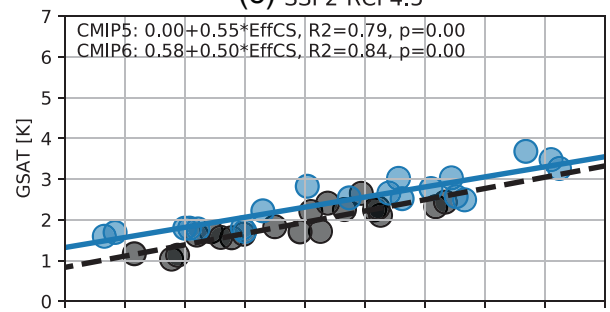

(f) SSP1-RCP2.6

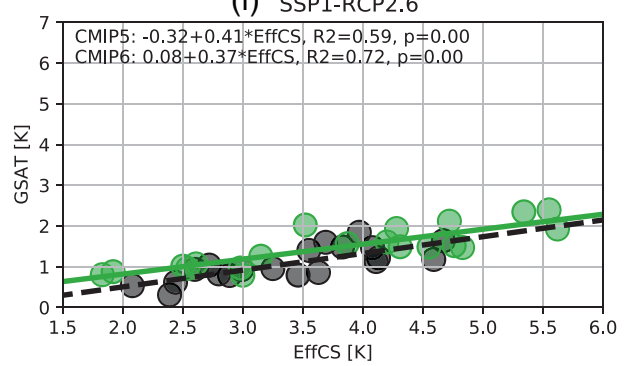

Figure 2. (a-c) GTE (m) and (d-f) GSAT (K) change (2081-2100 minus 1986-2005) of the CMIP6 (colored circles) and CMIP5 (black circles) models against their EffCS (K), together with least squares linear regression (CMIP6: colored, solid; CMIP5: black, dashed) against EffCS for SSP5-RCP8.5 (orange, (a, d)), SSP2-RCP4.5 (blue, (b, e)), and SSP1-RCP2.6 (green, (c, f)). The intercept, slope, $R$-squared and $p$-value of the regression are indicated in each panel. CMIP: Coupled Model Intercomparison Project; EffCS: effective climate sensitivity; GSAT: global mean surface air temperature; GTE:, global mean thermal expansion; SSP: shared socioeconomic pathway; RCP: representative concentration pathway.

Additionally, more of the intermodel variance of CMIP6 than of CMIP5 is explained by EffCS for all emissions scenarios, suggesting that the increased spread of EffCS causes the increased spread of GTE and GSAT change (Table S2). The correlations of both GTE and GSAT change with TCR are lower than with EffCS (Figure S4), consistent with previous CMIP5-based studies (Gregory et al., 2015; Grose et al., 2016, 2018). Thus, TCR is less useful than EffCS for characterizing GMSL projections.

\subsection{CMIP6-Based GMSL Projections}

Using the IPCC AR5 methods (Church et al., 2013) based on CMIP6 simulations results in ensemble-median projections of GMSL rise of 0.76, 0.56, and $0.47 \mathrm{~m}$ in 2100 (see Table S4 for 2081-2100 and Table S5 for a component breakdown) for SSP5-RCP8.5, SSP2-RCP4.5, and SSP1-RCP2.6, respectively (Figures 3a-3c). This represents a modest increase of 2,3 , and $3 \mathrm{~cm}(+3 \%,+6 \%$, and $+7 \%)$ relative to CMIP5. The 5\%-95\% range shifted up from $0.52-0.98,0.36-0.71$, and $0.28-0.61 \mathrm{~m}$ to $0.52-1.05,0.38-0.76$ and $0.30-0.64 \mathrm{~m}$. For the 95th percentile, this is an increase of 7,5 , and $3 \mathrm{~cm}(+7 \%,+7 \%$, and $+5 \%)$. In comparison, the projected rates of GMSL rise (average rates during 2095-2100) increase more: 1.4, 0.9, and $0.4 \mathrm{~mm} / \mathrm{yr}$ for the medians $(+12 \%,+15 \%$, and $+9 \%)$ and $3.5,1.6$, and $0.5 \mathrm{~mm} / \mathrm{yr}$ for the 95 th percentiles $(+21 \%,+18 \%$, and $+8 \%)$, respectively. Projections with the methods of Palmer et al. (2020) show a comparable increase from CMIP5 to CMIP6 (Figure S6). Thus, updating existing GMSL projections with CMIP6 data affects projections at 2100 only moderately but has a more pronounced impact beyond 2100 . 
(a) SSP5-RCP8.5

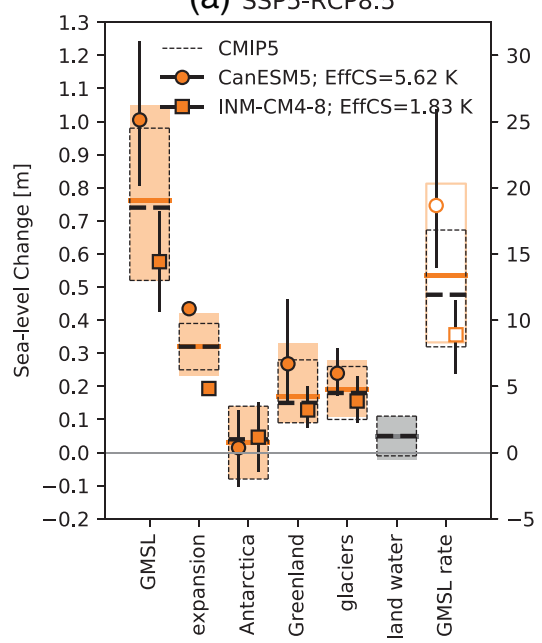

(d)

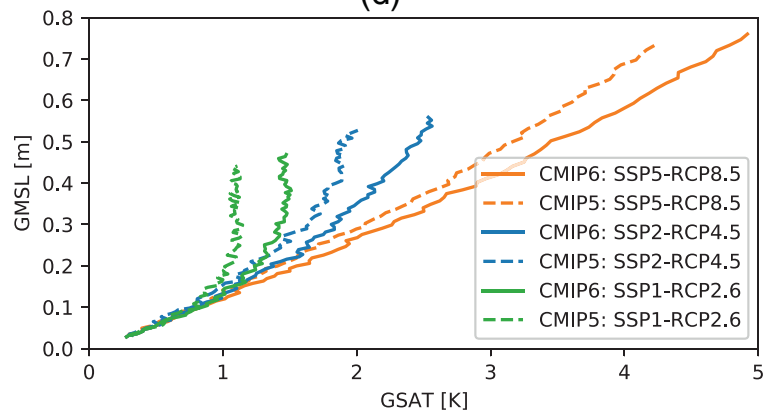

(b) SSP2-RCP4.5
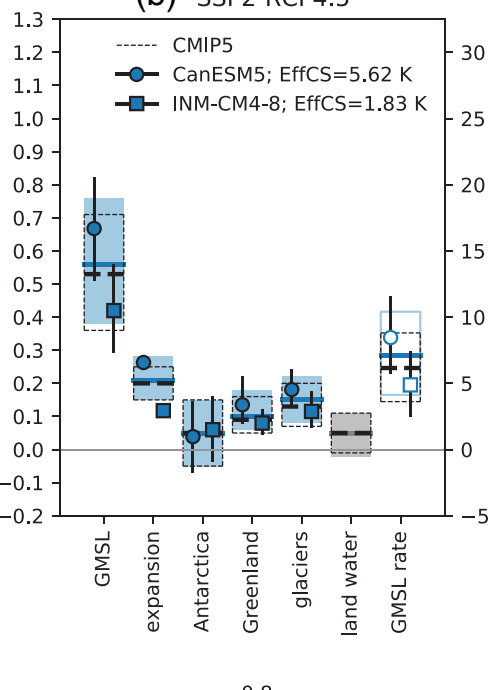

(c) SSP1-RCP2.6

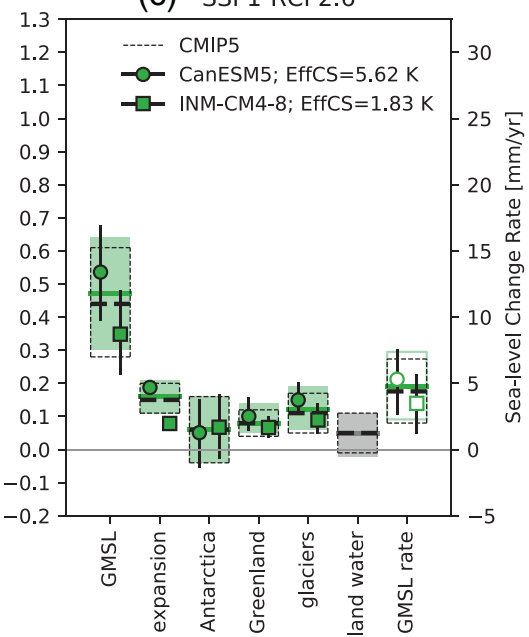

(e)

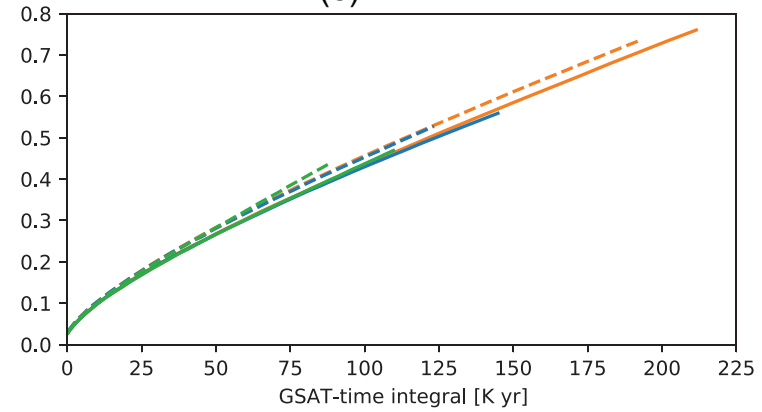

Figure 3. CMIP6-based projections of GMSL change and components in 2100 relative to 1986-2005 (m) and the average rate during 2095-2100 (mm/yr) computed using IPCC AR5 methods for (a) SSP5-RCP8.5 (orange), (b) SSP2-RCP4.5 (blue), and (c) SSP1-RCP2.6 (green), overlaid by CMIP5-based projections (Church et al., 2013, their Table 13.SM.1), and the ensemble-median GMSL change (m) against (d) ensemble-median GSAT (K) and (e) time-integrated ensemble-median GSAT change [K yr] for CMIP6 (solid curves) and CMIP5 (dashed curves) for 2007-2100 for the same emissions scenarios. The colored solid lines and boxes in $(\mathrm{a}-\mathrm{c})$ represent the CMIP6 ensemble-medians and 5\%-95\% ranges, and the black dashed lines those for CMIP5. The symbols and whiskers represent the median and 5\%-95\% for the two CMIP6 models with the highest and lowest EffCS, respectively. Note the secondary $y$-axes for the average rate of GMSL change. CMIP: Coupled Model Intercomparison Project; EffCS: effective climate sensitivity; GMSL, global mean sea-level; GSAT: global mean surface air temperature; IPCC AR5: fifth Assessment Report of the Intergovernmental Panel on Climate Change; SSP, shared socioeconomic pathway; RCP, representative concentration pathway.

To study intermodel differences, we also generate GMSL projections based on the 20 individual CMIP6 models directly (explained in Section 2.3 \& Text S1). The 5\%-95\% range for individual models can be substantially outside the CMIP6 ensemble 5\%-95\% range (Figure S7) and may be used to describe the outer envelope of GMSL projections instead. Large intermodel differences exist: the CMIP6 model with the highest EffCS (CanESM5; Swart et al., 2019) projects 5\%-95\% ranges of 0.81-1.24, 0.51-0.82, and 0.39-0.67 m for SSP5-RCP8.5, SSP2-RCP4.5, and SSP1-RCP2.6, respectively, whereas the model with the lowest EffCS (INM-CM4-8; Volodin \& Gritsun, 2018) projects 0.43-0.73, 0.29-0.56, and 0.23-0.48 m (Figures 3a-3c).

The differences between the 20,000-member ensemble consisting of the individual model projections combined $(20 \times 1,000)$ and the 450,000-member ensemble of GMSL projections constructed by sampling from GTE and GSAT change (Text S1) are mostly below the random uncertainty of the Monte Carlo method. However, the 5\%-95\% range of the thermal expansion of the 20,000-member ensemble is around $2 \mathrm{~cm}$ lower because the GTE distribution of CMIP6 is negatively skewed (Figures 1a-1c). Thus, the assumptions made in constructing the 450,000-member ensemble (i.e., that GTE and GSAT change are normally distributed and perfectly correlated to one another) are reasonable but imperfect. The actual correlation structure (Figure S5) can be preserved by directly sampling from the CMIP6 ensemble, but the number of models is likely insufficient for a proper representation. 
For both CMIP5 and CMIP6, the relationship between the median projection of GMSL and GSAT change becomes less linear toward 2100 under most scenarios (Figure 3d). In contrast, GMSL change and the time-integrated (cumulative sum of) GSAT change have a nearly linear and approximately scenario-independent relationship toward 2100 (Figure 3e). We explain why and discuss the implications for mitigation in the next section.

\section{Discussion \& Conclusions}

The increased EffCS in CMIP6 translates into substantially higher (e.g., 0.82, 0.56, $0.41 \mathrm{~K}$ for the medians) projections of 21st-century GSAT change than in CMIP5 for SSP5-RCP8.5, SSP2-RCP4.5, and SSP1-RCP2.6, consistent with previous findings (Forster et al., 2020; Tokarska et al., 2020). However, the differences between CMIP5- and CMIP6-based GMSL projections are relatively modest. Updating the IPCC AR5 projections with CMIP6 input leads to 5\%-95\% ranges of $0.52-1.05,0.38-0.76$, and $0.30-0.64 \mathrm{~m}$ in 2100 for SSP5-RCP8.5, SSP2-RCP4.5, and SSP1-RCP2.6. The medians have increased by $2-3 \mathrm{~cm}$ only and the 95th percentiles by $3-7 \mathrm{~cm}$ (see Table $\mathrm{S} 4$ for projections of other studies that used IPCC AR5 methods). This is caused by multiple different factors.

First, a higher GSAT increase results in a lower Antarctic GMSL contribution through increased snowfall accumulation. Moreover, the total land-ice contribution to GMSL is a (nearly linear) function of time-integrated GSAT change, since the rate of ice mass loss from the Greenland and Antarctic ice sheets and glaciers is related to GSAT change (Text S1). Since the relative difference in time-integrated GSAT change between CMIP5 and CMIP6 is smaller than the relative difference in GSAT change itself, the relative difference in GMSL rise in 2100 is also smaller. This additionally explains why projected GMSL rise rates around 2100 increase considerably from CMIP5 to CMIP6 (Figures 3a-3c), implying that GMSL projections beyond 2100 (e.g., Nauels et al., 2017; Palmer et al., 2018b, 2020) will be affected more strongly by increased EffCS. It also implies that time-integrated GSAT change up to 2100 is a better and more scenario-independent descriptor of total GMSL rise in 2100 than GSAT change in 2100. The approximate proportionality between the rate of OHU and GSAT change (Gregory et al., 2015; Gregory \& Mitchell, 1997) further contributes to this. To minimize the time-integral of GSAT change, emissions should be reduced early (e.g., Mengel et al., 2018), making time-integrated emissions metrics more relevant for GMSL rise targets than endpoint metrics (Collins et al., 2020; Olivié \& Peters, 2013).

Second, GTE is not substantially higher in CMIP6 than in CMIP5 and correlates with EffCS less strongly than GSAT change (Section 3.2). Given the increase in GSAT change, our results suggests that the average OHU efficiency (e.g., Kuhlbrodt \& Gregory, 2012) in CMIP6 is lower than in CMIP5. While the reasons for this are unclear (it could reflect increases in mean ocean stratification or changes in the patterns of projected surface warming), the intermodel differences in OHU efficiency may be dominated by the intermodel differences in the representation of ocean circulation processes (Newsom et al., 2020). Also, larger differences in GTE may only emerge after 2100, given the slow response of the deep ocean (Geoffroy \& Saint-Martin, 2013; Held et al., 2010).

Third, the effect of increased EffCS in some CMIP6 models on differences between CMIP5 and CMIP6 is partially balanced by the very low EffCS in others, such as INM-CM4-8 and INM-CM5-0. Thus, the lower end of the CMIP6 EffCS distribution also needs to be scrutinized. Additionally, the 20 models in our CMIP6 ensemble come from only 12 different modeling centers. Consequently, model weighting based on model interdependence (e.g., Brunner et al., 2020) may influence our results.

For consistency between GTE and GSAT change (Text S1), we only used CMIP6 models that provide simulations of both GTE and GSAT. Since the ensemble-mean GSAT change of all 31 CMIP6 models providing GSAT simulations is 0.11-0.27 K lower than that of the 20-member CMIP6 ensemble (Table S3), our GMSL projections might change when GTE simulations from additional models become available. Alternatively, for these models GTE could be derived from ocean temperature simulations (e.g., Lorbacher et al., 2015; Melet \& Meyssignac, 2015), or by emulation with simple climate models (e.g., Geoffroy \& Saint-Martin, 2013; Good et al., 2013; Palmer et al., 2018b). 
Although the EffCS range of CMIP6 lies partially outside the range recently assessed by the World Climate Research Program (WCRP) (Sherwood et al., 2020), increased EffCS may result from an improved representation of the atmosphere in at least some of the CMIP6 models (e.g., Bjordal et al., 2020; Gettelmann et al., 2019; Williams et al., 2020). Even though the differences in GMSL projections for 2100 between CMIP5 and CMIP6 are modest, the uncertainty around what constitutes EffCS in GCMs combined with the interest of risk-averse stakeholders in low-probability/high-impact GMSL projections (Hinkel et al., 2019; Stammer et al., 2019) clearly motivates exploring the outer envelope of GMSL projections by premising projections on individual CMIP6 GCMs (Figures 3a-3c \& S7).

If the real world would behave as simulated with CanESM5 (highest EffCS of CMIP6: 5.62 K), we cannot exclude a GMSL rise of $1.24 \mathrm{~m}$ for SSP5-RCP8.5 with more than 95\% confidence based on IPCC AR5 methods. This is $51 \mathrm{~cm}$ more than for INM-CM4-8 (lowest EffCS of CMIP6: $1.83 \mathrm{~K}$ ). Incorporating marine ice cliff instability in Antarctica in our projections may further augment this difference (Vega-Westhoff et al., 2020). Thus, the choice between characterizing the central part of the probability distribution and more comprehensively sampling the high end of the GMSL projection space, through subsetting the CMIP6 ensemble using EffCS, has a substantial impact on GMSL projections. Our results underline the need to constrain EffCS, GTE and GSAT in global climate models in order to better characterize uncertainty in sea-level projections.

\section{Data Availability Statement}

The CMIP6 data was extracted from ESGF on November 2, 2020. The authors thank Chris Jones for providing the CMIP6 GSAT time series processed using JASMIN on November 1, 2020. The CMIP6 data and Monte Carlo GMSL projections produced for this manuscript are available in the 4TU Data Repository: http://doi.org/10.4121/12958079.

\section{Acknowledgments}

The authors acknowledge the WCRP, which, through its Working Group on Coupled Modeling, coordinated and promoted CMIP6. The authors thank the climate modeling groups for producing and making available their model output, the ESGF for archiving the data and providing access, and the multiple funding agencies who support CMIP6 and ESGF. JG, MP and MR were supported by the Met Office Hadley Center Climate Program funded by the BEIS and Defra, UK.

\section{References}

Andrews, T., Gregory, J. M., Webb, M. J., \& Taylor, K. E. (2012). Forcing, feedbacks and climate sensitivity in CMIP5 coupled atmosphere-ocean climate models. Geophysical Research Letters, 39, 1-7. https://doi.org/10.1029/2012GL051607

Bjordal, J., Storelvmo, T., \& Carlsen, T. (2020). Equilibrium climate sensitivity above 5C plausible due to plausible due to state-dependent cloud feedback. Nature Geoscience, 13, 718-721. https://doi.org/10.1038/s41561-020-00649-1

Bodas-Salcedo, A., Mulcahy, J. P., Andrews, T., Williams, K. D., Ringer, M. A., Field, P. R., \& Elsaesser, G. S. (2019). Strong Dependence of Atmospheric Feedbacks on Mixed-Phase Microphysics and Aerosol-Cloud Interactions in HadGEM3. Journal of Advances in Modeling Earth Systems, 11, 1735-1758. https://doi.org/10.1029/2019MS001688

Brunner, L., Pendergrass, A. G., Lehner, F., Merrifield, A. L., Lorenz, R., \& Knutti, R. (2020). Reduced global warming from CMIP6 projections when weighting models by performance and independence. Earth System Dynamics, 11, 995-1012. https://doi.org/10.5194/ esd-11-995-2020

Church, J. A., Clark, P. U., Cazenave, A., Gregory, J. M., Jevrejeva, S., Levermann, A., et al. (2013). Sea level change. In T. F. Stocker, et al. (Eds.), In: Climate change 2013: The physical science basis. Contribution of working group I to the fifth assessment Report of the intergovernmental Panel on climate change. Cambridge University Press, Cambridge, United Kingdom.

Collins, W. J., Frame, D. J., Fuglestvedt, J. S., \& Shine, K. P. (2020). Stable climate metrics for emissions of short and long-lived speciescombining steps and pulses. Environmental Research Letters, 15, (2), 024018. http://dx.doi.org/10.1088/1748-9326/ab6039

Dangendorf, S., Marcos, M., Wöppelmann, G., Conrad, C. P., Frederikse, T., \& Riva, R. (2017). Reassessment of 20th century global mean sea level rise. Proceedings of the National Academy of Sciences, 114, 5946-5951. https://doi.org/10.1073/pnas.1616007114

DeConto, R. M., \& Pollard, D. (2016). Contribution of Antarctica to past and future sea-level rise. Nature, 531, 591-597. https://doi. org/10.1038/nature17145

Edwards, T. L., Brandon, M. A., Durand, G., Edwards, N. R., Golledge, N. R., Holden, P. B., et al. (2019). Revisiting Antarctic ice loss due to marine ice-cliff instability. Nature, 566, 58-64. https://doi.org/10.1038/s41586-019-0901-4

Eyring, V., Bony, S., Meehl, G. A., Senior, C. A., Stevens, B., Stouffer, R. J., et al. (2016). Overview of the Coupled Model Intercomparison Project Phase 6 (CMIP6) experimental design and organization. Geoscientific Model Development, 9, 1937-1958. https://doi.org/10.5194/ gmd-9-1937-2016

Forster, P. M., Andrews, T., Good, P., Gregory, J. M., Jackson, L. S., \& Zelinka, M. (2013). Evaluating adjusted forcing and model spread for historical and future scenarios in the CMIP5 generation of climate models. Journal of Geophysical Research Atmospheres, 118, 1139-1150. https://doi.org/10.1002/jgrd.50174

Forster, P. M., Maycock, A. C., McKenna, C. M., \& Smith, C. J. (2020). Latest climate models confirm need for urgent mitigation. Nature Climate Change, 19, 7-10. https://doi.org/10.1038/s41558-019-0660-0

Frederikse, T., Jevrejeva, S., Riva, R. E. M., \& Dangendorf, S. (2018). A consistent sea-level reconstruction and its budget on basin and global scales over 1958-2014. Journal of Climate, 31, 1267-1280. https://doi.org/10.1175/JCLI-D-17-0502.1

Geoffroy, O., \& Saint-Martin, D. (2013). Transient climate response in a two-layer energy-balance model. Part I: Analytical solution and parameter calibration using CMIP5 AOGCM experiments. Journal of Climate, 26, 1841-1857. https://doi.org/10.1175/JCLI-D-12-00195.1

Gettelmann, A., Hannay, C., Bacmeister, J. T., Neale, R. B., Pendergrass, A. G., Danabasoglu, G., et al. (2019). High climate sensitivity in the community earth system model version 2 (CESM2). Geophysical Research Letters, 2, 8329-8337. https://doi.org/10.1029/2019GL083978 
Good, P., Gregory, J. M., \& Lowe, J. A. (2011). A step-response simple climate model to reconstruct and interpret AOGCM projections. Geophysical Research Letters, 38, 1-5. https://doi.org/10.1029/2010GL045208

Good, P., Gregory, J. M., Lowe, J. A., \& Andrews, T. (2013). Abrupt CO2 experiments as tools for predicting and understanding CMIP5 representative concentration pathway projections. Climate Dynamics, 40, 1041-1053. https://doi.org/10.1007/s00382-012-1410-4

Gregory, J. M., Andrews, T., \& Good, P. (2015). The inconstancy of the transient climate response parameter under increasing CO2. Philosophical Transactions of the Royal Society A, 373(2054), 20140417. http://dx.doi.org/10.1098/rsta.2014.0417

Gregory, J. M., Griffies, S. M., Hughes, C. W., Lowe, J. A., Church, J. A., Fukimori, I., et al. (2019). Concepts and terminology for sea level: Mean variability and change, both local and global. Surveys in Geophysics, 40, 1251-1289. https://doi.org/10.1007/s10712-019-09525-z

Gregory, J. M., Ingram, W. J., Palmer, M. A., Jones, G. S., Stott, P. A., Thorpe, R. B., et al. (2004). A new method for diagnosing radiative forcing and climate sensitivity. Geophysical Research Letters, 31, 2-5. https://doi.org/10.1029/2003GL018747

Gregory, J., \& Lowe, J. (2000). Predictions of global and regional sea-level rise using AOGCMs with and without flux adjustment. Geophysical Research Letters, 27, 3069-3072. https://doi.org/10.1029/1999GL011228

Gregory, J. M., \& Mitchell, J. F. B. (1997). The dimate response to CO2 of the Hadley Center coupled AOGCM with and without flux adjustment. Geophysical Research Letters, 24, 1943-1946. https://doi.org/10.1029/97GL01930

Grose, M. R., Colman, R., Bhend, J., \& Moise, A. F. (2016). Limits to global and Australian temperature change this century based on expert judgment of climate sensitivity. Climate Dynamics, 48, 3325-3339. https://doi.org/10.1007/s00382-016-3269-2

Grose, M. R., Gregory, J., Colman, R., \& Andrews, T. (2018). What climate sensitivity index is most useful for projections? Geophysical Research Letters, 45, 1559-1566. https://doi.org/10.1002/2017GL075742

Held, I., Winton, M., Takahasi, K., Delworth, T., Zeng, F., \& Vallis, G. K. (2010). Probing the fast and slow components of global warming by returning abruptly to pre-industrial forcing. Journal of Climate, 23, 2418-2427. https://doi.org/10.1175/2009JCLI3466.1

Hinkel, J., Church, J. A., Gregory, J. M., Lambert, E., Le Cozannet, G., Lowe, J., et al. (2019). Meeting user needs for sea level rise information: A decision analysis perspective. Earth's Future, 7, 320-337. https://doi.org/10.1029/2018EF001071

Hobbs, W., Palmer, M. D., \& Monselesan, D. (2016). An energy conservation analysis of ocean drift in the CMIP5 global coupled models. Journal of Climate, 29, 1639-1653. https://doi.org/10.1175/JCLI-D-15-0477.1

Kopp, R. E., Horton, R. M., Little, C. M., Mitrovica, J. X., Oppenheimer, M., Rasmussen, D. J., et al. (2014). Probabilistic 21st and 22nd century sea-level projections at a global network of tide-gauge sites. Earth's Future, 2, 383-406. https://doi.org/10.1002/2014EF000239

Kuhlbrodt, T., \& Gregory, J. M. (2012). Ocean heat uptake and its consequences for the magnitude of sea level rise and climate change. Geophysical Research Letters, 39, 1-6. https://doi.org/10.1029/2012GL052952

Levermann, A., Winkelmann, R., Nowicki, S., Fastook, J. L., Frieler, K., Greve, R., et al. (2014). Projecting Antarctic ice discharge using response functions from SeaRISE ice-sheet models. Earth System Dynamics, 5, 271-293. https://doi.org/10.5194/esd-5-271-2014

Lorbacher, K., Nauels, A., \& Meinshausen, M. (2015). Complementing thermosteric sea level rise estimates. Geoscientific Model Development, 8, 2723-2734. https://doi.org/10.5194/gmd-8-2723-2015

Lyu, K., Zhang, X., Church, J. A., \& Hu, J. (2015). Quantifying internally generated and externally forced climate signals at regional scales in CMIP5 models. Geophysical Research Letters, 42, 9394-9403. https://doi.org/10.1002/2015GL065508

Meehl, G. A., Senior, C. A., Eyring, V., Flato, G., Lamarque, J., Stouffer, R. J., et al. (2020). Context for interpreting equilibrium climate sensitivity and transient climate response from the CMIP6 Earth system models. Science Advances, 6, 1-10. https://doi.org/10.1126/ sciadv.aba1981

Meinshausen, M., Smith, S. J., Calvin, K., Daniel, J. S., Kainuma, M. L. T., Lamarque, J.-F., et al. (2011). The RCP greenhouse gas concentrations and their extensions from 1765 to 2300. Climatic Change, 109, 210-241. https://doi.org/10.1007/s10584-011-0156-z

Melet, A., \& Meyssignac, B. (2015). Explaining the spread in global mean thermosteric sea level rise in CMIP5 climate models. Journal of Climate, 28, 9918-9940. https://doi.org/10.1175/JCLI-D-15-0200.1

Mengel, M., Nauels, A., Rogelj, J., \& Schleussner, C.-F. (2018). Committed sea-level rise under the Paris Agreement and the legacy of delayed mitigation action. Nature Communications, 9, 1-10. https://doi.org/10.1038/s41467-018-02985-8

Nauels, A., Meinshausen, M., Mengel, M., Lorbacher, K., \& Wigley, T. M. L. (2017). Synthesizing long-term sea level rise projections - the MAGICC sea level model v2.0. Geoscientific Model Development, 10, 2495-2524. https://doi.org/10.5194/gmd-10-2495-2017

Newsom, E., Zanna, L., Khatiwala, S., \& Gregory, J. M. (2020). The influence of warming patterns on passive ocean heat uptake. Geophysical Research Letters, 47, 71-75. https://doi.org/10.1029/2020GL088429

Nicholls, R. J., \& Cazenave, A. (2010). Sea-level rise and its impact on coastal zones. Science, 328, 1517-1520. https://doi.org/10.1126/ science. 1185782

Nijsse, F. J. M. M., Cox, P. M., \& Williamson, M. S. (2020). Emergent constraints on transient climate response (TCR) and equilibrium climate sensitivity (ECS) from historical warming in CMIP5 and CMIP6 models. Earth System Dynamics, 11, 737-750. https://doi. org/10.5194/esd-11-737-2020

Olivié, D. J. L., \& Peters, G. P. (2013). Variation in emission metrics due to variation in $\mathrm{CO} 2$ and temperature impulse response functions. Earth System Dynamics, 4, 267-286. https://doi.org/10.5194/esd-4-267-2013

Oppenheimer, M., Glavovic, B. C., Hinkel, J., van de, Wal, R., Magnan, A. K., Abd-Elgawad, A., et al. (2019). Sea level rise and implications for low lying islands, coasts and communities. In H.-O. Pörtner, et al. (Eds.), IPCC special report on the ocean and cryosphere in a changing climate.

O'Neill, B. C., Kriegler, E., Riahi, K., Ebi, K. L., Hallegatte, S., Carter, T. R., et al. (2014). A new scenario framework for climate change research: the concept of shared socioeconomic pathways. Climatic Change, 122, 387-400. https://doi.org/10.1007/s10584-013-0905-2

Palmer, M. D., Gregory, J. M., Bagge, M., Calvert, D., Hagedoorn, J. M., Howard, T., et al. (2020). Exploring the drivers of global and local sea-level change over the 21st century and beyond. Earth's Future, 8, e2019EF001413. https://doi.org/10.1029/2019EF001413

Palmer, M. D., Harris, G. R., \& Gregory, J. M. (2018). Extending CMIP5 projections of global mean temperature change and sea level rise due to thermal expansion using a physically-based emulator. Environmental Research Letters, 13(8), 084003. https://doi. org/10.1088/1748-9326/aad2e4

Palmer, M., Howard, T., Tinker, J., Lowe, J., Bricheno, L., Calvert, D., et al. (2018). UKCP18 marine report.

Rugenstein, M., Bloch-Johnson, J., Gregory, J., Mauritsen, T., Li, C., Frolicher, T. L., et al. (2020). Equilibrium climate sensitivity estimated by equilibrating climate models. Geophysical Research Letters, 47(4), e2019GL083898. https://doi.org/10.1029/2019GL083898

Sen Gupta, A., Jourdain, N. C., Brown, J. N., \& Monselesan, D. (2013). Climate drift in CMIP5 models. Journal of Climate, 26, 8597-8615. https://doi.org/10.1175/JCLI-D-12-00521.1

Sherwood, A. S., Webb, M. J., Annan, J. D., Armour, K. C., Forster, P. M., Hargreaves, J. C., et al. (2020). An assessment of Earth's climate sensitivity using multiple lines of evidence. Review of Geophysics, 58, 1-166. https://doi.org/10.1029/2019RG000678 
Slangen, A. B. A., Carson, M., Katsman, C. A., van de Wal, R. S. W., Koehl, A., Vermeersen, L. L. A., \& Stammer, D. (2014). Projecting twenty-first century regional sea-level changes. Climatic Change, 124, 317-332. https://doi.org/10.1007/s10584-014-1080-9

Slangen, A. B. A., Katsman, C. A., van de Wal, R. S. W., Vermeersen, L. L. A., \& Riva, R. E. M. (2012). Toward regional projections of twenty-first century sea-level change based on IPCC SRES scenarios. Climate Dynamics, 38, 1191-1209. https://doi.org/10.1007/ s00382-011-1057-6

Stammer, D., Van De Wal, R. S. W., Nicholls, R. J., Church, J. A., Le Cozannet, G., Lowe, J. A., et al. (2019). Framework for high-end estimates of sea level rise for stakeholder applications. Earth's Future, 7, 923-938. https://doi.org/10.1029/2019EF001163

Swart, N. C., Cole, J. N. S., Kharin, V. V., Lazare, M., Scinocca, J. F., Gillett, N. P., et al. (2019). The Canadian Earth system model version 5 (CanESM5.0.3). Geoscientific Model Development, 12, 4823-4873. https://doi.org/10.5194/gmd-12-4823-2019

Taylor, K., Stouffer, R. J., \& Meehl, G. A. (2012). An overview of CMIP5 and the experiment design. Bulletin of the American Meteorological Society, 93, 485-498. https://doi.org/10.1175/BAMS-D-11-00094.1

Tokarska, K. B., Stolpe, M. B., Sippel, S., Fischer, E. M., Smith, C. J., Lehner, F., \& Knutti, R. (2020). Past warming trend constrains future warming in CMIP6 models. Science Advances, 6, 1-14. https://doi.org/10.1126/sciadv.aaz9549

Vega-Westhoff, B., Sriver, R. L., Hartin, C., Wong, T. E., \& Keller, K. (2020). The role of climate sensitivity in upper-tail sea level rise projections. Geophysical Research Letters, 47, 1-8. https://doi.org/10.1029/2019GL085792

Voldoire, A., Saint-Martin, D., Sénési, S., Decharme, B., Alias, A., Chevallier, M., et al. (2019). Evaluation of CMIP6 DECK experiments with CNRM-CM6-1. Journal of Advances in Modeling Earth Systems, 11, 2177-2213. https://doi.org/10.1029/2019MS001683

Volodin, E. M., \& Gritsun, A. S. (2018). Simulation of possible future climate changes in the 21st century in the INM-CM5 climate model. Earth System Dynamics, 9, 1235-1242. https://doi.org/10.5194/esd-9-1235-2018

Williams, K. D., Hewitt, A. J., \& Bodas-Salcedo, A. (2020). Use of short-range forecasts to evaluate fast physics processes relevant for climate sensitivity. Journal of Advances in Modeling Earth Systems, 12, 1-9. https://doi.org/10.1029/2019MS001986

Zelinka, M. D., Myers, T. A., Mccoy, D. T., Po-chedley, S., Caldwell, P. M., Ceppi, P., et al. (2020). Causes of higher climate sensitivity in CMIP6 models. Geophysical Research Letters, 47, 1-12. https://doi.org/10.1029/2019GL085782 